ISSN 2409-2665

Journal of Logistics, Informatics and Service Science

Vol. 8 (2021) No. 2, pp. 119-133

DOI:10.33168/LISS.2021.0207

\title{
An Optimized Two-Phase Approach Based on Social Spider Optimization for Retinal Vascular Segmentation in Fundus Images
}

\author{
Hameed AlQAheri \\ Department of Information System and Operation Management, College of \\ Business Administration, Kuwait University, Kuwait; hameed.alqaheri@ku.edu.kw \\ hameed.alqaheri@ku.edu.kw
}

\begin{abstract}
It is time-consuming and tedious for ophthalmologists to go through every fundus photo and diagnose it. Accurate and efficient segmentation of retinal vessels from fundus images represents a key step in diagnosing and screening many relevant diseases. It is a prerequisite for computer-aided diagnosis of eye diseases. This paper proposes a two-phase approach based on lateral inhibition neural networks and social spider optimization for retinal vascular segmentation in fundus images. It uses two publicly available image databases. The first one contains one hundred high-quality fundus images obtained from the Armed Forces Institute of Ophthalmology (AFIO), Pakistan and the second one is DRIVE database. It was assessed based on specificity, sensitivity, and accuracy and the analysis of the obtained results shows the means to be $96.645 \%, 79.239 \%$, and $96.47 \%$, respectively, and the average of the statistical power is $96 \%$. The Gravitational Force (GF) and Lateral Inhibition Neural networks are applied to enhance the images before segmentation, and the determination of the threshold is then solved by reducing the cross-entropy between the image and its segmented image. Optimal multi-threshold selection using social spider optimization for selecting the optimal threshold values is applied. The results show that the proposed approach achieves better color maintenance and contrast enhancement. The proposed approach also leads to significant features and low contrast improvement and brightness preservation in the enhanced image while maintaining the natural feel of the original image and allowing the detection of more vessels and better segments.
\end{abstract}

Keywords: Hypertensive retinopathy, contrast enhancement, vessel segmentation, social spider optimization, gravitational force, lateral inhibition network 


\section{Introduction}

Over the past few years, many clinical studies and research have been conducted using fundus images to automate the process of pathology examination and diagnosis. Many diseases can be examined and diagnosed using fundus retinal images (Chalakkal et al., 2020; Franklin and Rajan, 2014). Retinal imaging technology offers a non-invasive method for observing retinal vessels, and because of its low cost and reproducibility, it has been widely used in clinical applications (Fadzil and Izhar., 2011; Aranya and Selvarani, 2016). However, low-quality fundus images result in uncertainty in clinical follow-up, leading to a risk of misdiagnosis. However, because of the special beam of light for imaging the fundus and the structure of the retina, natural image enhancement methods cannot be used directly to address this issue (Shen and Fu, 2020; Salazar-Gonzalez et al., 2014; Geetha and Balasubramanian, 2016). Recently, the use of computer-aided diagnostic (CAD) systems is rapidly increasing, which helps in the early detection and accurate analysis of retinal fundus (Porwal et al., 2018). Vascular segmentation of retinal fundus images can be used as a CAD system to diagnose various retinal diseases. For example, Eman Abdel Maksoud and Mohammed Elmogy in (Maksoud and Elmogy, 2020) proposed a comprehensive diagnostic system for early signs and different degrees of diabetic retinopathy using fundus retinal images based on detecting pathological changes. The system achieved good results (up to $92.8 \%$ ) in terms of positive predictive value.

Automatic and accurate fundus images segmentation is an extremely important task in computer-aided diagnosis (CAD) in analyzing medical images to diagnose diseases such as glaucoma, hypertensive retinopathy, and diabetic retinopathy (HR) (Intaramanee et al., 2016; Nagpal and Malarvel, 2021; Wong and McIntosh, 2005). As one of the most important anatomical structures of the retina, blood vessels are analyzed to diagnose many diseases, such as retinopathy, obstruction, and other diseases that threaten vision. Vascular segmentation can also be a pre-processing step for segmenting other retinal structures, such as the optic disc, microvasculature, and fovea, (Furtado et al., 2017; Feng et al., 2007; Singh and Srivastava, 2016). This paper proposes a two-phase approach based on lateral inhibition neural networks and social spider optimization for retinal vascular segmentation in fundus images. This process relies on careful adjustment of image pre-processing so that blood vessels can be detected. First, the fundus image quality is enhanced based on the Gravity Force Law (GFL) and lateral inhibition neural networks (LINN). The retinal vessel is, then, segmented for further diagnosis based on the multi-thresholding approach, and then the social spider optimizer is used to select the best thresholding values.

\section{The main contributions of the paper are:}

- Gravity Force Law (GFL) and lateral inhibition neural networks (LINN) are applied to enhance the retinal fundus images before the segmentation.

- A spider social optimization algorithm is applied to the segmentation of the vessel images to obtain better segmentation results. 
- The threshold selection is solved by reducing the cross-entropy values between the image and its segmented image.

The remainder of this paper is structured as follows. In Section (2) a brief explanation of the basic methods used is presented. Section 3 discusses the main phases of the proposed automated system for hypertensive retinopathy detection. Section 4 discusses the obtained results. Finally, Section 5 concludes the paper.

\section{Preliminaries}

This section briefly explains the basic methods used in this study, including the Gravity Force Law (GFL), lateral inhibition neural networks (LINN), the minimum cross-entropy thresholding, and the social spider optimization algorithm with some of the key basic concepts. A more comprehensive review can be found in the sources as (Katırcıŏglu et al., 2019; Li and Le, 1993; Sonali et al., 2019).

\subsection{Lateral Inhibition Neural Network (LINN)}

Every object attracts other objects with the strength of gravity based on Newton's law (Sonali et al., 2019). The GF between the two objects is inversely proportional to the square of the distance between the two objects and directly proportional to their masses, and is defined as shown in Equation (1) (Oliva et al., 2019).

$$
F=G \frac{M 1 \cdot M 2}{R^{2}}
$$

where $F$ is the gravitational force in Newton, $\mathrm{M}_{1}$ and $\mathrm{M}_{2}$ are masses, $\mathrm{G}$ is the gravity constant, and $\mathrm{R}$ represents the distance between two objects.

In Limulus visual physiological electrical investigations, the hypothesis of lateral network inhibition was established, and each eye of Limulus is considered as one receptor. The stimulability of the receptor is reduced when the receiver (receptor) receives a strong light alarm. This is considered to be a phenomenon of lateral inhibition networks. Assuming that the emissions of light forces are $e_{A}$ and $e_{B}$ for two receivers $A$ and $B$ and they are lighted. In the simultaneous illumination of $A$ and $B$, the emission light forces are reduced accordingly to $\mathrm{f}_{\mathrm{A}}$ and $\mathrm{f}_{\mathrm{B}}$. The inhibition of receptor of $A$ by receptor of $B$ and vice versa (receptor of $A$ by receptor of $B$ ) is responsible for this change. Eqs. (2) and (3) mathematically define the lateral inhibitory effect (Sonali et al., 2019).

$$
\begin{gathered}
e_{A}=f_{A}-k_{B A}\left(f_{B}-f_{A B}\right) \\
e_{B}=f_{B}-k_{A B}\left(f_{A}-f_{B A}\right)
\end{gathered}
$$

To be used in image processing, the LINN needs to be extended to two dimensions where $k_{A B}$ and $k_{B A}$ indicate the coefficients for lateral inhibition of the two receptors, while $f_{A B}$ and $f_{B A}$ refer to the lateral inhibition threshold value. By omitting the threshold value, the classic lateral inhibition technique is used as a nonrepetitive LINN model (Sonali et al., 2019). 


$$
\operatorname{Out}(x, y)=\operatorname{In}(x, y)-\sum_{i=-1}^{l} \sum_{j=-1}^{l} k(i, j) \cdot \operatorname{In}(x+i, y+j)
$$

where $\operatorname{In}(\mathrm{x}, \mathrm{y})$ and $\operatorname{Out}(\mathrm{x}, \mathrm{y})$ are the input image and the output image respectively, and $\mathrm{k}(\mathrm{i}, \mathrm{j})$ is the inhibition coefficient matrix where the inhibition area is equal to 1 . The typical LINN is noise sensitive and frequently combined with median filtering to remove noise. Therefore, Equation (4) can be assumed as follows:

$$
\begin{gathered}
\operatorname{Out}(\mathrm{x}, \mathrm{y})=\overline{\operatorname{In}(\mathrm{x}, \mathrm{y})}-\sum_{\mathrm{i}=-\mathrm{l}}^{\mathrm{l}} \sum_{\mathrm{j}=-1}^{\mathrm{l}} \mathrm{k}(\mathrm{i}, \mathrm{j}) \cdot \operatorname{In}(\mathrm{xi}, \mathrm{y} \mathrm{j}) \\
\overline{\operatorname{In}(\mathrm{x}, \mathrm{y})}=\underset{(r, s) \in\left(-l^{\prime}, l^{\prime}\right)}{\text { median filter }}[\operatorname{In}(\mathrm{K} 1+\mathrm{r}, \mathrm{K} 2+\mathrm{s})],
\end{gathered}
$$

$\overline{\operatorname{In}(\mathrm{x}, \mathrm{y})}$ is the median filter value of the input image, and $l^{\prime}$ is the size of the median filtering window.

\subsection{The minimum cross-entropy thresholding approach}

Cross entropy was developed by Kullback (Katırcioğlu et al., 2019). Let $P=$ $\left\{\mathrm{p}_{1}, \mathrm{p}_{2}, \mathrm{p}_{3}, \ldots, \mathrm{p}_{\mathrm{n}}\right\}$ and $\mathrm{Q}=\left\{\mathrm{q}_{1}, \mathrm{q}_{2}, \mathrm{q}_{3}, \ldots, \mathrm{q}_{\mathrm{n}}\right\}$ be two distribution probability functions on the same set. The cross-entropy between $P$ and $Q$ is defined as (6):

$$
P(P, Q)=\sum_{i=1}^{n} p_{i} \log \frac{p_{i}}{q^{i}}
$$

The approach selects a number of thresholds by minimizing the cross-entropy between the original and segmented images. Let $\mathrm{I}(\mathrm{x}, \mathrm{y})$ be the original image, and $\mathrm{h}(\mathrm{i}), \mathrm{i}=1,2, . . \mathrm{L}$, and $\mathrm{L}$ is the number of gray levels. Then, the segmented image, denoted by It , is constructed using $\mathrm{t}$ as the threshold value as shown in the following Equation (7):

$$
-I_{t}(x, y)=\left\{\begin{array}{ll}
\mu(1, t) & I<t \\
\mu(t, L+1) & I \geq t
\end{array}\right\}
$$

Where $\mu$ indicates the mean of the original image intensity.

\subsection{Social Spider Optimization (SSO)}

SSO is a swarm intelligence-based metaheuristic algorithm ( $\mathrm{Li}$ and Le, 1993). The SSO imitates the behavior of spiders. Spiders determine the position of the prey through vibration on the spider web. Any unusual vibration allows the social spider to move towards the source of vibration to search for food. The search area of the SSO was explored with a chain-like social spider structure. Each insect determines the direction of the food through signals analyzed from the vibrations generated from the spider web. In the first iteration, several social spiders were inserted into a spider web. The spider population size (PS) parameter identifies the search agents (i.e., number of spiders). All spiders interacted with each other to determine the best food source location. Each spider uses a mask $m$ of length $D$ that consists of a $0-1$ binary vector where $D$ is the variables number searched. Each parameter in mask $m$ is changed to imitate the social spider random-walk operation. The vibration intensity at $\mathrm{x}$ is calculated using Equation (8): 


$$
\mathrm{I}(\mathrm{x}, \mathrm{x}, \text { iter })=\log \left(\frac{1}{\mathrm{~F}(\mathrm{x})-\mathrm{C}}+1\right)
$$

Where $\mathrm{C}$ is a constant.

The iteration attenuation is calculated by Equation (9):

$$
\mathrm{I}\left(\mathrm{x}_{1}, \mathrm{x}_{2}, \text { iter }\right)=\mathrm{I}\left(\mathrm{x}_{1}, \mathrm{x}, \text { iter }\right) \times \exp \left(-\frac{\mathrm{D}\left(\mathrm{x}_{1}, \mathrm{x}_{2}\right)}{\sigma \times \mathrm{r}_{\mathrm{a}}}\right)
$$

Where $\mathrm{D}\left(\mathrm{x}_{1}, \mathrm{x}_{2}\right)$ denotes the distances between $\mathrm{x}_{1}$ and $\mathrm{x}_{2}$. The standard deviation of all members along one searched dimension is represented by $\sigma$ and the free parameter is represented by $r_{a}$.

\section{The proposed automated system for hypertensive retinopathy detection}

The proposed automated blood vessel segmentation approach comprises the following two fundamental phases: (1) the retina enhancement phase in which fundus image enhancement algorithms based on lateral inhibition neural networks (LINNs) AND Newton's gravitational force law are presented. It is adopted and used to improve the quality of the fundus images and make the feature segmentation phase more reliable; and (2) the vessel segmentation phase in which the cross-entropy with the social spider optimization algorithm for vessel segmentation is used.

The two phases of the system are presented in detail in the following section (refer to Figure 1).

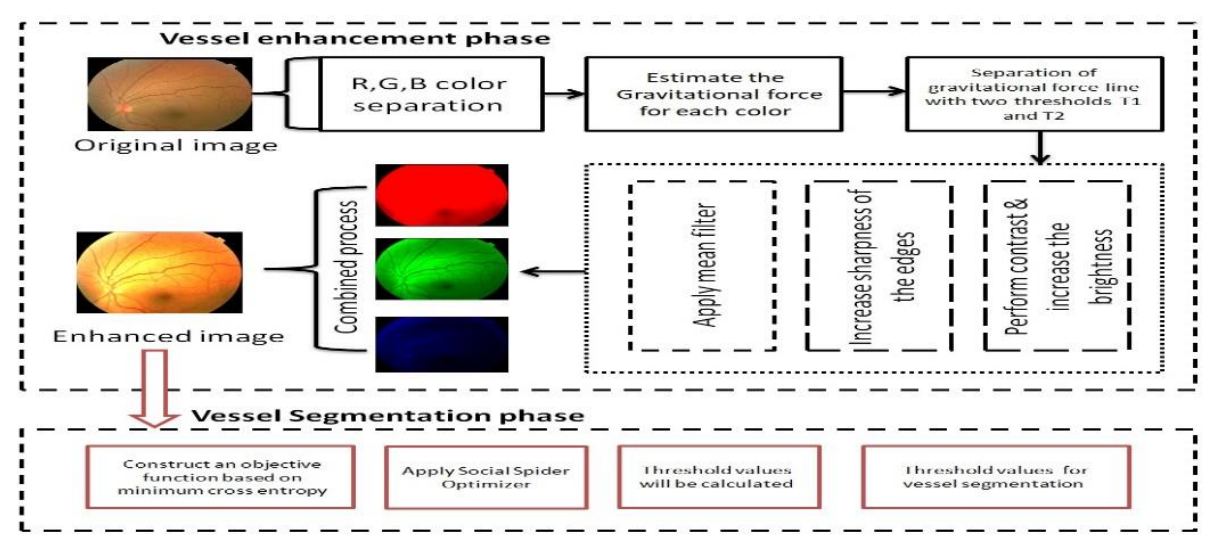

Fig. 1: The General Architecture of the Vessel Segmentation Model

\subsection{Gravitational Force based Retinal Enhancement Phase}

The enhancement process aims to minimize noise from the color fundus images in every dimension, modify the brightness and contrast, and efficiently expose information of the edges and regions of the retinal image. The gravitational force law is used for each color $(\mathrm{R}, \mathrm{G}, \mathrm{B})$ dimension in the color image space to build information measures in the lateral inhibition neural network (LINN). Contrast adjustment, noise reduction, and edge clarification are conducted on these 
information measures after being subjected to two threshold values $(C)$. The full steps of this process are illustrated in Figure 2, and the main steps are outlined as follows:

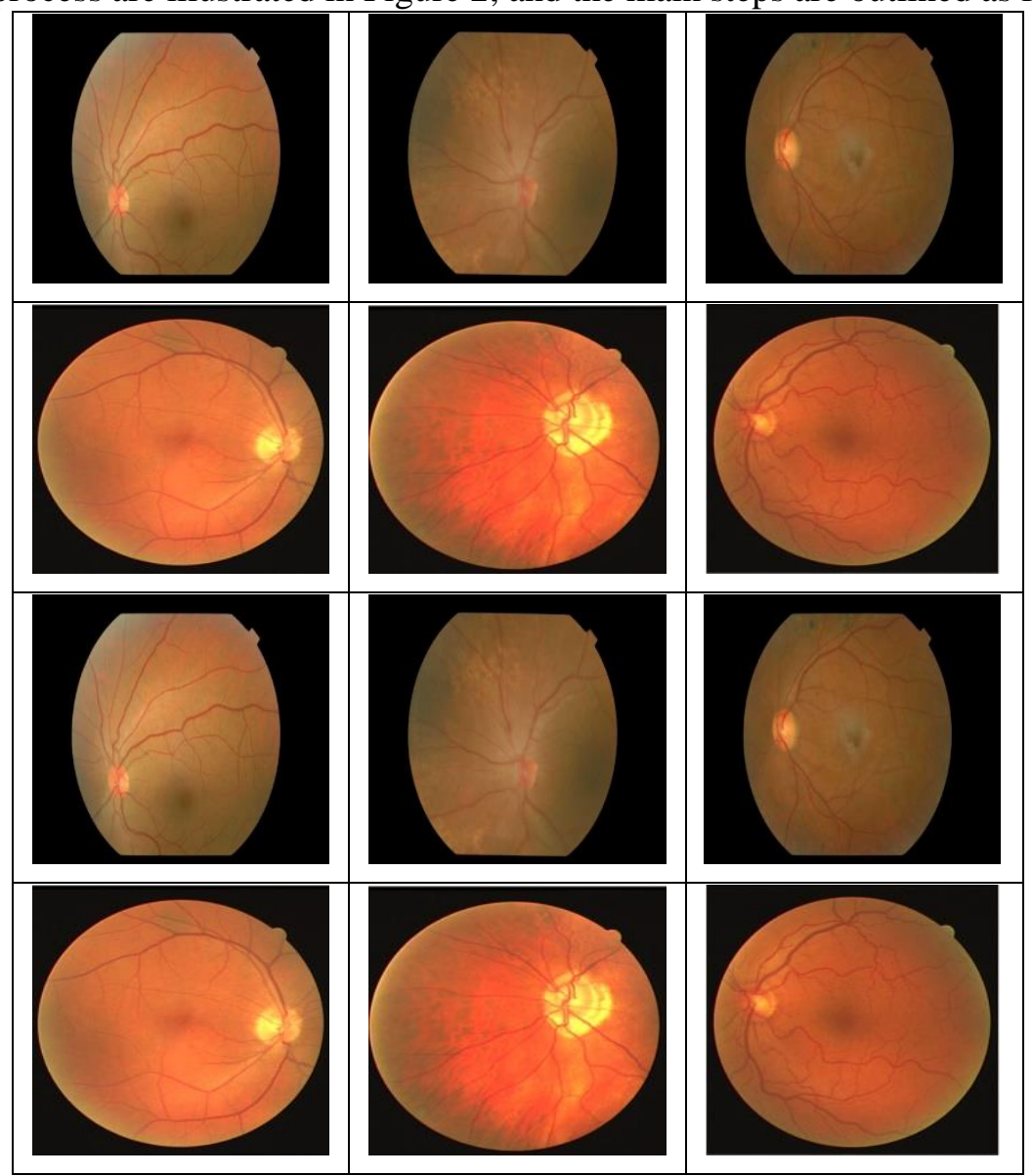

Fig. 2: Samples of the fundus images data - first raw from 2nd raw (DRIVE DATABASE) (Kumar and Radhika, 2019)

1. Divide the vessel image into two-dimensional pictures of red, green, and blue.

2. Estimate the gravitational force with nearby pixels for each color with the center pixel with a mask of $3 \times 3$ using Equation (10).

$$
f_{-} \text {sum_ } X_{i}=\sum_{j=1}^{i} F X_{i j}
$$

3. Where $X_{i}$ represents the three colors $\mathrm{R}, \mathrm{B}$, and $\mathrm{G}$

4. The three resulting total gravity force matrices, red, green, and blue, are then display the two threshold values $\left(T_{1}\right.$ and $\left.T_{2}\right)$ in the second stage. The process is based on the LINN that takes place when the data on gravitational strength is within the $T_{1}$ and $T_{2}$ range.

a. Apply a median filter in case the $T_{1}<f_{-} s u m_{-} X_{i}$ 


$$
O(m, n)_{X}=\frac{1}{9} \sum_{j=-1}^{1} \sum_{i=-1}^{1} \operatorname{In}(m+i, n+j)_{X}
$$

Where $\operatorname{In}(m+j, n+i)_{X}$ represents the original image for each color $\mathrm{x}(\mathrm{R}, \mathrm{G}, \mathrm{B})$

Else

b. Increase the sharpness of the edges when $T_{1}<f_{-}$sum_ $X_{i}<T_{2}$ using the following Equation, which is based on LINN.

$$
O(m, n)_{X}=\text { Median filter }-\sum_{i=-1}^{1} \sum_{j=-1}^{1} K(i, j) . f_{-} \operatorname{sum}(m+i, n+j)-{ }_{x}
$$

Where $k(i, j)$ is coefficient constant

Else

c. Perform contrast and increase the brightness

$$
O(m, n)_{X}=\text { Median filter } * c \sum_{i=-1}^{1} \sum_{j=-1}^{1} f_{-} \operatorname{sum}(m+i, n+j)--x
$$

where $\mathrm{c}$ is a coefficient constant and the contrast amount in the area is defined by its relation to pixels within the specified window. Then, the RGB output retinal fundus image is obtained by combining all three-color images in one color space.

\subsection{Multilevel Threshold Vessel Segmentation Phase}

This section discusses the main steps of the proposed social spider algorithm for determining the optimal threshold values for image segmentation. This is applied to the resulting image of the enhancement phase. The main objective of the spider optimizer is, then, to reduce the statistical criteria of the cross-entropy that is used as an objective function of the social spider optimizer using Equation 12, which iterates until the cut-off criterion is reached by evaluating the cross-entropy function and until the set of thresholds that get the minimum value is found ( $\mathrm{Yu}$ and $\mathrm{Li}, 2015$ ). The cross-entropy objective function can be written as follows:

$$
\begin{gathered}
f(t)=\sum_{i=1}^{t_{i}-1} i h(i) \log \left(\mu\left(1, t_{1}\right)\right)-\sum_{i=t_{1-1}}^{L} i h(i) \log \left(\mu\left(t_{1}, L+1\right)\right) \\
=-K^{1}\left(1, t_{1}\right) \log \frac{k^{1}\left(1, t_{1}\right)}{k^{0}\left(1, t_{1}\right)}-K^{1}\left(t_{1}, L+1\right) \log \frac{k^{1}\left(t_{1, L+1}\right)}{k^{0}\left(t_{1, L+1)}\right)}
\end{gathered}
$$

where $k^{1}, k^{0}$ are the zero-moment point and the first-moment point $\mathrm{k}, \quad t=$ $\left\{t_{1}, t_{2}, t_{3}, \ldots \ldots, t_{n}\right\}$ are the set of thresholds, and $n$ represents the number of thresholds that exist, where $t_{1}<t_{2}<t_{3}<\cdots . .<t_{n}$. Finally, the objective function of the minimum cross-entropy for multilevel thresholding is defined as follows (11):

$$
f(t)=K^{1}\left(t_{i-1}, t\right) \log \frac{k^{1}\left(t_{i-1}, t\right)}{k^{0}\left(t_{i-1}, t\right)}
$$




\section{Results and Discussion}

Fundus publicly available image databases: The proposed model is tested using publicly available image databases that contain one hundred high-quality fundus images obtained from the Armed Forces Institute of Ophthalmology (AFIO), Rawalpindi, Pakistan and DRIVE database (Kumar and Radhika, 2019). The database contains detailed annotations of retinal vascular patterns, arterioles, and venules making it possible to calculate the arteriovenous ratio, optic nerve head area, and other retinal abnormalities such as scleral exudates and cotton spots. Figure 3 shows samples of fundus image data.

Eye fundus image enhancement results: To evaluate the obtained imageenhanced results, the following measures are used: These are contrast, edge intensity, and sharpness (Katırcıoğlu et al., 2019). Where

$$
\begin{gathered}
\text { Contrast }=\sqrt{\frac{1}{n(n-1)}\left(n \sum g_{i j}^{2}-\left(\sum g_{i j}\right)^{2}\right)} \\
\text { Edge intensity }=\sum_{\mathrm{ij}}\left|\left[\begin{array}{c}
1 \\
-1
\end{array}\right] * g_{i j}\right| \\
\text { Sharpness }=\sum_{\mathrm{ij}}\left|\left[\begin{array}{ccc}
0 & -1 & 0 \\
-1 & 4 & -1 \\
0 & -1 & 0
\end{array}\right] * \mathrm{~g}_{\mathrm{ij}}\right|
\end{gathered}
$$

where $\mathrm{n}$ is the number of pixel values in the fundus image, and $\mathrm{g}$ is the pixel value. The normalized cross-correlations (NCC) and structural similarity index (SSI) factors were utilized for every enhanced eye fundus image.

Table 1. The setting parameters values of the fundus images enhancement experiments.

\begin{tabular}{|c|c|}
\hline Parameter & Setting value \\
\hline gravity constant $(\mathrm{G})$ & 10 \\
\hline values $T_{1}$ and $T_{2}$ & 0.01 and 0.73 \\
\hline k coefficient & 1.4 \\
\hline local contrast adjustment & 0.373 \\
\hline
\end{tabular}

Table 1 presents the setting parameter values of the fundus image enhancement experiments. Figure 3 shows the performance of the enhanced eye fundus images based on contrast, edge intensity, and sharpness measures, and it can be seen that their values are evident. Also, the SSI values $(00.84,0.7942,0.8284$, and 0.8118$)$ for the selected samples indicate how close the enhanced retina fundus images are compared to the original one. The high NCCs values $(1.4,1.5068,1.4464$, and 1.5274) show fewer lighting changes on the improved images than the input original retina fundus images. This indicates that the pixel values of the original images did not change significantly during the filtering process. The contrast process guaranteed that the focus in the dark values (in the Green and Blue dimensions) was extended to a large range, and the bright red levels (in $\mathrm{R}$ ) shifted to a smooth state during the contrast 
stage.

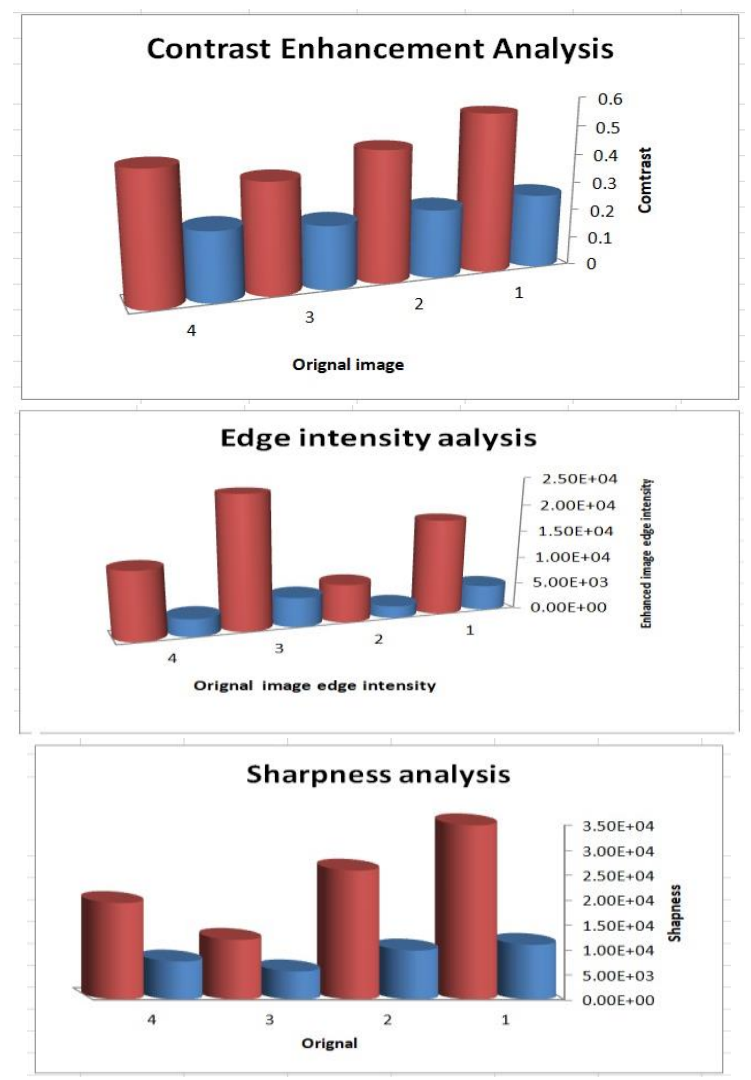

Fig. 3: The evaluation results for eye fundus enhanced image results in terms of contrast, edge intensity, sharpness

Vessel segmentation results: Specificity (Spe), Sensitivity (Sen), and Accuracy (Acc) are three measures used to analyze and obtain the right degree of vessel retina segmentation. These are defined as follows:

$$
\begin{gathered}
\text { Spe }=\frac{T_{N}}{T_{N}+F_{p}} \\
\text { Sen }=\frac{T_{p}}{T_{p}+F_{N}} \\
\text { Acc }=\frac{T_{p}+T_{N}}{T_{p}+T_{N}+F_{p}+F_{N}}
\end{gathered}
$$

Where True positive $\left(T_{p}\right)$ represents the amount of the pixel, as a vessel, in both images (ground truth and segmented vessel images).True negative $\left(T_{N}\right)$ represents the pixel number correctly categorized as a non-vessel image (ground truth and segmented vessel images).False-positive $\left(F_{p}\right)$ represents the amount of the pixel as a vessel in the segmented vessel images and not as a vessel in the ground truth image. False-negative $\left(F_{N}\right)$ represents the pixel number incorrectly categorized as not being a vessel in the image of the fragmented vessel as it is matched with the vessel in the 
image of reality on the ground.

Figure 4 shows the segmentation results compared with the ground truth images. Figure 4 (a) and (b) show the original and enhancement results, respectively, and (c) represents the ground thruth and (d) shows the segmentation resultsl. It can be observed that the contrast of the images is improved and the information about the vessel and region of the eye fundus images is clearer. Information on vessel segmentation in the fundus image is highlighted.

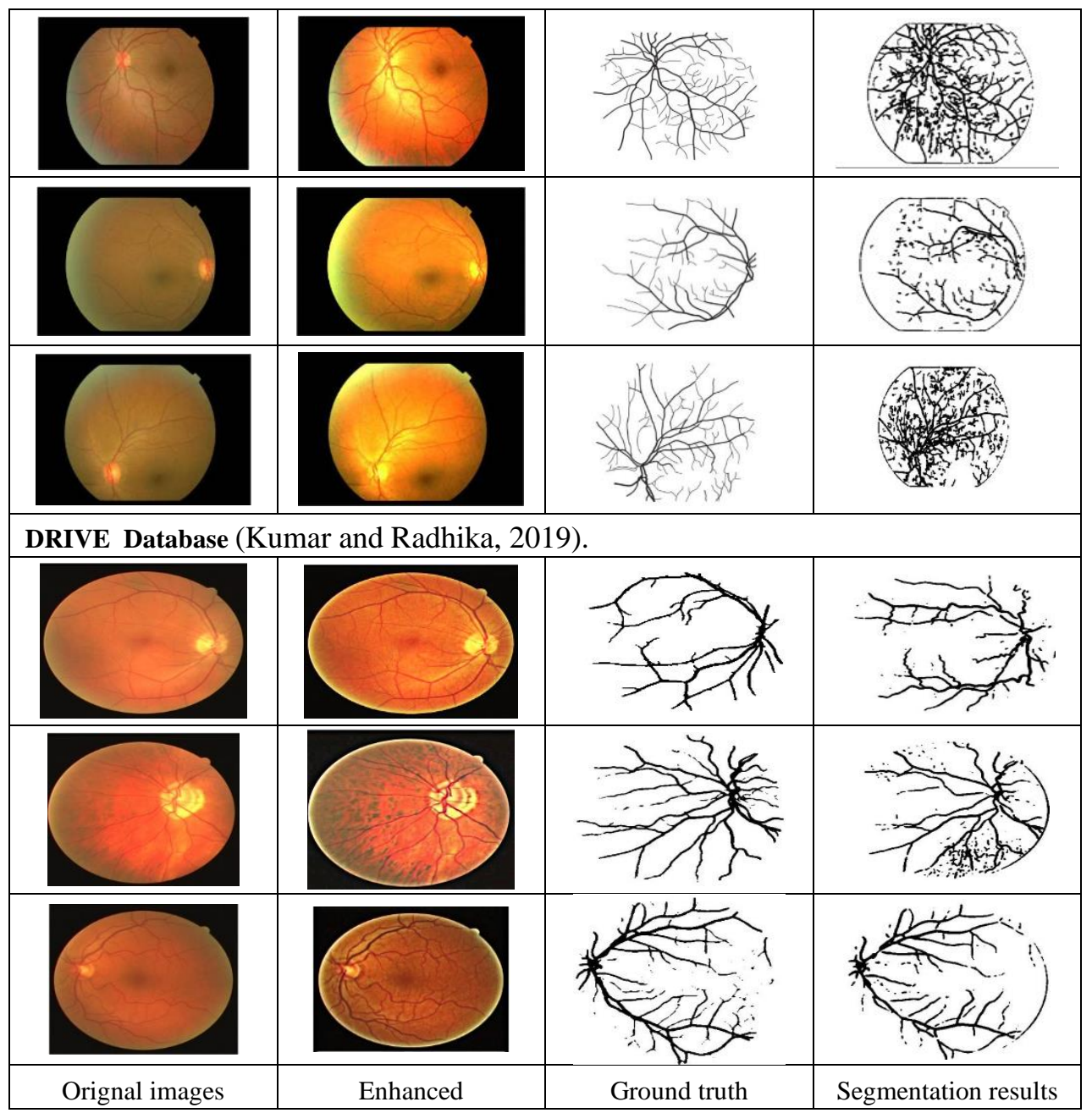

Fig. 4: The vessel segmentation results

Table 2 shows the performance vessel segmentation results based on the three metrics above (Spe, Sen, and Acc). The specificity, sensitivity, and accuracy means are $96.645 \%, 79.239 \%$, and $96.47 \%$, respectively. The statistical power is $96 \%$ as an average when the segmented vessel image is compared with the segmented images of the segmented vessel. The minimum Specificity value is $93.28 \%$ in image number 
6 , and the maximum number of specificity is $98.72 \%$ in image number 11 .

Table 2. Results of the vessel segmentation of fundus Images in terms of sensitivity, specificity, and accuracy.

\begin{tabular}{|c|c|c|c|}
\hline Image\# & Specificity (Spe) & Sensitivity (Sen) & Accuracy (Acc) \\
\hline Image1 & $96 \%$ & $50 \%$ & $91.3 \%$ \\
\hline Image2 & $95.03 \%$ & $62.36 \%$ & $93.07 \%$ \\
\hline Image3 & $98.12 \%$ & $42.43 \%$ & $95.69 \%$ \\
\hline Image 4 & $94.37 \%$ & $52.74 \%$ & $92.2 \%$ \\
\hline Image 5 & $98.34 \%$ & $81.85 \%$ & $94.11 \%$ \\
\hline Image 6 & $93.28 \%$ & $78.36 \%$ & $97.34 \%$ \\
\hline Image 7 & $97.11 \%$ & $53.22 \%$ & $91.70 \%$ \\
\hline Image 8 & $95.03 \%$ & $65.92 \%$ & $98.03 \%$ \\
\hline Image 9 & $98.64 \%$ & $82.11 \%$ & $93.44 \%$ \\
\hline Image 10 & $93.73 \%$ & $79.04 \%$ & $96.09 \%$ \\
\hline Image11 & $98.72 \%$ & $89.14 \%$ & $98.11 \%$ \\
\hline Image12 & $97.95 \%$ & $83.81 \%$ & $97.03 \%$ \\
\hline Avarage & $96.645 \%$ & $79.239 \%$ & $96.47 \%$ \\
\hline
\end{tabular}

Table 3 indicates further statistical analysis and interpretation of the eye fundus diagnostic based on the ratio of sensitivity and specificity, including False positive rate (FPR), False negative rate (FNR), Power (P), Positive likelihood ratio (PLR), and negative likelihood ratio (NLR). Likelihoods ratios are used to assess the benefit of a particular probable diagnostic test and the likely that a patient will be affected (PLR and (NLR).

The statistical power $(\mathrm{P})$ is when an experiment finds a positive result, assuming that the alternative hypothesis is true. This is simply the opposite result to a false negative, hence the statistical power equals $1-\mathrm{FNR}$. It is fairly standard to design experiments to choose sample sizes. The obtain statistical power, in this case, is $96 \%$ (as an average).

From Table 4, it can be observed that the accuracy rate of the proposed model proves that optimization is a better choice in carrying out the segmentation process. Despite the different data sets used, the maturity of the DRIVE data sets, and the limitation of the new data sets in terms of their size, however, that we have used for the first time, several challenges we have face that need further investigation and analysis.

Table 3. Fundus diagnostic and statistical analysis

\begin{tabular}{|c|c|c|c|c|c|}
\hline Image\# & $\begin{array}{c}\text { FPR=1- } \\
\text { Spec }\end{array}$ & $\begin{array}{c}\text { FNR=1- } \\
\text { Sen }\end{array}$ & $\begin{array}{c}\text { Power }=1- \\
\text { FNR }\end{array}$ & $\begin{array}{c}\text { PLR=sen/(1- } \\
\text { spec })\end{array}$ & $\begin{array}{c}\text { NLR=(1 - sen } \\
) / \text { spec }\end{array}$ \\
\hline
\end{tabular}




\begin{tabular}{|c|c|c|c|c|c|}
\hline 1 & $4 \%$ & $50 \%$ & $96 \%$ & 12.5 & 0.520833 \\
\hline 2 & $5 \%$ & $38 \%$ & $95 \%$ & 12.5472837 & 0.396085 \\
\hline 3 & $2 \%$ & $58 \%$ & $98 \%$ & 22.56914894 & 0.586731 \\
\hline 4 & $6 \%$ & $47 \%$ & $94 \%$ & 9.367673179 & 0.500795 \\
\hline 5 & $2 \%$ & $18 \%$ & $98 \%$ & 49.30722892 & 0.184564 \\
\hline 6 & $7 \%$ & $22 \%$ & $93 \%$ & 11.66071429 & 0.23199 \\
\hline 7 & & $3 \% 47 \%$ & $97 \%$ & 18.41522491 & 0.481722 \\
\hline 8 & $5 \%$ & $34 \%$ & $95 \%$ & 13.26358149 & 0.358624 \\
\hline 9 & $1 \%$ & $18 \%$ & $99 \%$ & 60.375 & 0.181367 \\
\hline 10 & $6 \%$ & $21 \%$ & $94 \%$ & 12.60606061 & 0.223621 \\
\hline avarege & $4 \%$ & $35 \%$ & $96 \%$ & 22.2611916 & 0.366633 \\
\hline
\end{tabular}

Table 4. Comparative analysis

\begin{tabular}{|c|c|c|c|}
\hline Reference & Optimization algorithm & Accuracy & Data sets \\
\hline $\begin{array}{c}\text { https://data.mendeley.com/dat } \\
\text { asets/3csr652p9y/1 }\end{array}$ & $\begin{array}{c}\text { Ant Colony } \\
\text { Optimization }\end{array}$ & $94.07 \%$ & Drive \\
\hline Kumar and Radhika, 2019 & $\begin{array}{c}\text { Artificial Bee Colony } \\
\text { (ABC) }\end{array}$ & $96.35 \%$ & Drive \\
\hline $\begin{array}{c}\text { https://en.wikipedia.org/wiki/ } \\
\text { Newton\%27s_law_of_univers } \\
\text { al_gravitation }\end{array}$ & $\begin{array}{c}\text { Particle Swarm } \\
\text { Optimization }\end{array}$ & $96.18 \%$ & DRIVE \\
\hline The proposed Model & $\begin{array}{c}\text { Social Spider } \\
\text { Optimzation }\end{array}$ & $96.47 \%$ & $\begin{array}{c}\text { New data } \\
\text { sets }\end{array}$ \\
\hline
\end{tabular}

\section{Conclusion and future works}

This paper proposed a two-phase approach based on lateral inhibition neural networks and social spider optimization for retinal vascular segmentation in fundus images. The first phase is based on lateral inhibition neural networks (LINN) for noise removal and contrast enhancement for the first phase, followed by the vessel segmentation process by reducing the cross-entropy between the retina enhanced image and its segmented image with the optimal multi-threshold selection using social spider optimization to select the best threshold values. The proposed approach is assessed using several performance metrics, including contrast, edge intensity, and sharpness, to evaluate the enhancement results and specificity, sensitivity, and accuracy for analyzing the obtained segmentation results with an average of $96.645 \%$, $79.239 \%$, and $96.47 \%$, respectively. The results show that the proposed segmentation approach achieves better performance on balancing the contrast enhancement, conservation color distribution, and the vessel segmentation method detects more vessels and better segments. In a future research, the proposed work will be extended to segment the artery/vein in detecting vascular abnormalities applying deep learning technology-based semantic segmentation for artery/vein classification. 


\section{References}

Aranya, M., \& Selvarani, A. G. (2016). Fundus Image Screening for Diabetic Retinopathy. Indian J. Sci. Technol. 9(25), 1-6.

Chalakkal R. J., Waleed, H.A., \& Sheng, C.H.(2020). Fundus retinal image analyses for screening and diagnosing diabetic retinopathy, macular edema, and glaucoma disorders, Diabetes and Fundus OCT, 1: Computer-Assisted Diagnosis. ComputerAssisted Diagnosis. 59-111.

Cinsdikici, M. G., \& Aydın, D. (2009). Detection of blood vessels in ophthalmoscope images using MF/ant (matched filter/ant colony) algorithm. Computer Methods and Programs in Biomedicine. 96(2), 85-95.

Fadzil, A., \& Izhar, M.H, Nugroho, H. (2011). Analysis of retinal fundus images for grading of diabetic retinopathy severity. Med Biol Eng Comput. 49.2.693-700. https://doi.org/10.1007/s11517-011-0734-2.

Feng, P., Pan, Y., Wei, B., Jin, W., \& Mi, D. (2007). Enhancing retinal image by the Contourlet transform. Pattern Recognit. Lett. 28(4), 516-522.

Franklin, S. W., \& Rajan, S. E. (2014). Computerized screening of diabetic retinopathy employing blood vessel segmentation in retinal images. Biocybern. Biomed. Eng, 34(2).117-124.

Furtado, P., Travassos, C., Monteiro, R., Oliveira, S., Baptista, C., \& Carrilho, F. (2017). Segmentation of Eye Fundus Images by density clustering in diabetic retinopathy. in Biomedical \& Health Informatics (BHI), 2017 IEEE EMBS International Conference on. 25-28.

Geetha, R.R., \& Balasubramanian, L. (2016). Retinal blood vessel segmentation employing image processing and data mining techniques for computerized retinal image analysis. Biocybern Biomed Eng. 36(1), 102-18.

https://data.mendeley.com/datasets/3csr652p9y/1.

https://en.wikipedia.org/wiki/Newton\%27s_law_of_universal_gravitation.

Intaramanee, T., Rasmequan, S., Chinnasarn, K., Jantarakongkul, B., \& Rodtook, A. (2016). Optic disc detection via blood vessels origin using Morphological end point. in 2016 International Conference On Advanced Informatics: Concepts, Theory And Application (ICAICTA) 
Katırcıŏlu, F., Çay, Y., \& Cingiz, Z. (2019). Infrared image enhancement model based on gravitational force and lateral inhibition networks. Infrared Physics \& Technology. 100, 15-27.

Kavya K., Dechamma M.G., \& Santhosh Kumar, B.J. (2016). Extraction of Retinal Blood Vessel Using Artificial Bee-Colony Optimization. Journal of Theoretical and Applied Information Technology. 88(3), 535-540.

Kumar, N. C. S., \& Radhika, Y. (2019). Optimized Maximum Principal Curvature Based Segmentation of Blood Vessels from Retinal Images, Biomedical Research. An International Journal of Medical Sciences. 30(2).

Li, C.H., \& Le, C.K. (1993). Minimum cross entropy thresholding. Pattern Recognition. 26(4), 617-625.

Maksoud, E. A., \& Elmogy, M. (2020). A comprehensive diagnosis system for early signs and different diabetic retinopathy grades using fundus retinal images based on pathological changes detection. Computers in Biology and Medicine. 126, (1-23).

Nagpal, D. S. N. Panda., \& Malarvel, M. (2021). Hypertensive Retinopathy Screening through Fundus Images-A Review, 2021 6th International Conference on Inventive Computation Technologies (ICICT). 924-929.

Niemeijer, J.J., Staal, B.V., Ginneken, M., Loog, M.D., \& Abramoff, M.D. (2015). DRIVE: Digital Retinal Images for Vessel Extraction. http://www.isi.uu.nl/Research/Databases/DRIVE (2004). Online; Accessed at 6 Jan 2015.

Oliva, D., Hinojosa, S., \& Osuna-Enciso, V. et al. (2019). Image segmentation by minimum cross entropy using evolutionary methods. Soft Computing, 2019, 23(2), 431-450. https://doi.org/10.1007/s00500-017-2794.

Porwal, P., Pachade, S., Kokare, M., Deshmukh, G., \& Sahasrabuddhe, V. (2018). Automatic retinal image analysis for the detection of diabetic retinopathy. Biomedical signal and image processing in patient care. 146-61.

Salazar-Gonzalez, A., Kaba, D., Li, Y., \& Liu, X. (2014). Segmentation of the blood vessels and optic disk in retinal images, IEEE J. Biomed. Health Inform. 18(6), 18741886.

Shen, Z., \& Fu, H. (2020). Shen, J., Shao, L. Modeling and Enhancing Low-quality Retinal Fundus Images. 
Singh, N.P., \& Srivastava, R. (2016). Retinal blood vessels segmentation by using Gumbel probability distribution function based matched filter. Comput Methods Prog Biomed. 129, 40-50.

Sonali, S.S., Singh, A.K., Ghrera, S.P., \& Elhoseny, M. (2019). An approach for denoising and contrast enhancement of retinal fundus image using CLAHE. Opt Laser Technol. 110, 87-98.

Wong, T.Y., \& McIntosh, R. (2005). Hypertensive retinopathy signs as risk indicators of cardiovascular morbidity and mortality. British Medical Bulletin. 73(1), 57-70.

Yu, J. J.Q., \& Li, V. O.K. (2015). A social spider algorithm for global optimization, Applied Soft Computing. 30, 14-627. https://doi.org/10.1016/j.asoc.2015.02.014. 